\title{
Delivery of Engineered Therapeutics and Medication
}

\author{
Dong-an Wang
}

Received: 1 February 2011 / Accepted: 22 March 2011 / Published online: 14 April 2011

(C) Springer Science+Business Media, LLC 2011

The development of modern medicine has been broadening the denotation of therapeutic "drugs" from solely nonliving remedial matters made of natural or synthetic compounds toward various "living molecules," such as nucleic acids, and further toward living beings per se, such as viable cells. Nowadays, all these "drugs" - or, namely therapeutic agents — can be delivered to the targeted lesions in controllable manners with the aid of engineering strategies. As such, besides the efforts in discovery and development of new drugs and cures, studies on the delivery of engineered therapeutics and medication have come onto the stage and drawn great attention in the areas of biomedicine and bio-pharmacy.

Each therapeutic agent has its unique properties and specific requirements for the delivery platform in order to achieve the desired performance. The role of delivery vehicle is to protect the therapeutic agent, preventing it from any degradation or pre-mature release and yet still allowing its controlled release at the therapeutic dosage at the targeted site. Since choice of materials and platform design would directly determine the release profile and drug stability during the delivery process, a myriad of biomaterials have been explored and integrated into various platform designs in pursuit of a compatible delivery system for each therapeutic agent.

In this theme issue, the focus is on the current approaches in delivering two groups of therapeutic agents: bio-macro/molecules and cells. Balmayor and colleagues

D.-a. Wang $(\bowtie)$

Division of Bioengineering, School of Chemical and Biomedical

Engineering (SCBE), Nanyang Technological University (NTU)

Singapore, Singapore

e-mail: DAWang@ntu.edu.sg review the limitations of existing routes of drug administrations and also the up-to-date research progress in this field. In recent years, the role of scaffolds has been transformed from a mere cell vehicle to a multi-functional delivery system. Ji et al. provide an overview of the advancement and challenges in incorporating biological molecules into electrospun scaffold for tissue engineering applications, while Hu's group summarizes the different ways of integrating growth factor-carrying microspheres into fibrous scaffolds.

Medicinal delivery includes both "traditional" pharmaceutical products and nucleic acids. Pharmaceutical products range from natural or synthetic small molecules to biomacromolecules like growth factors. Nelson and colleagues describe the use of poly(ester urethane) urea (PEUU) scaffolds to achieve controlled release of growth factors. Lima and coworkers propose to use a superhydrophobic surface in the fabrication of protein encapsulated hydrogel beads.

In the delivery of nucleic acids, there are three articles focusing on DNA transportation. Falco et al. employ a polymeric scaffold as a release device for plasmid encoding for insulin-like growth factor and green fluorescent protein. They show that the porosity of scaffold alters the plasmid loading and release rate and that the scaffold is effective in transfecting myoblasts. Jiang and colleagues utilize string-like micellar nanoparticles to deliver plasmid DNA and demonstrate that this platform can mediate efficient transgene expression in liver. Ramasubramanian's team explores a combinatorial strategy which enables administration of multiple genes for osteogenesis in human adipose-derived stem cells.

Current nucleic acid manipulation is not limited to addition or up-regulation of gene expressions; gene silencing has attracted its fair share of attention in this field. Zhang and co-workers construct a dual-functioning lentiviral vectors- 
suppressing Type-I collagen expression in synovium-derived mesenchymal stem cells for chondrogenesis. In the area of nonviral delivery, Dong et al. report successful localized release of antisense oligonucleotides reducing tumor necrosis factoralpha (TNF- $\alpha)$ from a cationic gelatin-chitosan copolymer hydrogel and hence repressing osteoclastogenesis in vivo. Mahato and colleagues silence IKK- $\alpha$ gene, a recently discovered mediator of inflammation and metastasis in prostate cancer, and show that there is a reduction in migration and invasion in prostate cancer cells. Mountziaris and co-workers use poly(DL-lactic-co-glycolic acids) (PLGA)based microparticles to achieve sustained release of antiinflammatory siRNA over 28 days, targeted for treating temporomandibular joint (TMJ) inflammation.

As cell-based therapy brings new opportunities in current clinical treatment, administration of stem cells as "living drugs" becomes an essential part of therapeutics. Before cell transplantation, the first step in cell-based therapy is to harvest the stem cells and expand them in vitro to attain sufficient cell numbers for intended tissue replacement or repair. Bramono's group finds that there is a favorable effect of bone marrow stromal-derived heparan sulfate in the ex vivo expansion of hematopoietic stem cells. Hwang and colleagues describe the strategy of chondrogenic priming of human adipose-derived mesenchymal stem cells so as to overcome the shortage of primary chondrocytes in tissue regeneration. Wang et al. set up a hyaluronic acid-based hydrogel embedded with growth factor-releasing microspheres to mimic the niche for neural stem cell growth and maintenance.

After rigorous cell expansion in vitro, cells are to be delivered to the target site to fulfill the therapeutic objectives. The carrier chosen in cell delivery plays a pivotal role in determining the efficacy of the therapy. Waterhouse and co-workers modify the plasma-activated coated surface with recombinant human tropoelastin and show that there is retention of cell-binding moieties even after exposure to supraphysiological concentrations of the major coagulation enzymes. Lin's team highlights the issue of how physical properties of the carrier material, such as mesh size of the hydrogel, can affect cellular activities in the encapsulated chondrocytes. Su et al. develop an open hollow-shell structure that enhances anchorage-dependent cell loading efficiency and at the same time provides room for cell growth and extracellular matrix secretion.

In summary, these experts update us with the current status and future prospects of medicinal and cellular administration together with exquisite designs of delivery platforms and manipulation of cells. We would like to thank all invited authors for their contributions made with great efforts and standards. We believe that the papers gathered in this theme issue will be an enormous asset to everyone working in the field of therapeutic and medicinal delivery.

\section{INTERVIEW WITH DR. DONG-AN WANG}

What do you think holds the key to your success as a biomaterials/ tissue-engineering scientist?

Ever since I began my research life when I was a graduate student over 10 years ago, it has always been my dream to be a successful biomaterialist/tissue engineer. I would not define my current status as a "success" but rather at one of the few milestones in the career. I believe that in order to succeed in this inter-disciplinary area, one must have good knowledge in different subjects, including chemistry and material science as well as some biology and preclinical sciences; at the same time, one should also have his/her own specialty in a certain area.

\section{What do you consider to be your key research accomplishments?}

We have developed bioresponsive hydrogels/hydrogelcomposites for therapeutic cell delivery. We aimed to create significant room inside the hydrogel bulk to overcome the problem of space constraint in most scaffold systems. This strategy not only preserves the hydrogel's advantage of injectability and controls the fate of delivered cells, but it also provides space for cell proliferation and endogenous extracellular matrix secretion. We have applied gene/antisense delivery in engineered regenerative medicine and showed that desirable genes could be expressed while undesirable genes could be down-regulated at the same time to facilitate functional tissue regeneration. We have applied adjacent reproducible tissue-sourced stem cells, namely synovium-derived mesenchymal stem cells (SMSCs), for engineered therapeutics. The SMSCs have multipotency in musculoskeletal regeneration for cartilage, bones, tendons, and muscles. SMSGs have better proliferation ability and superiority in chondrogenesis, which make them a good candidate for cartilage tissue regeneration. We have also shown that tissue-biomaterial integration could be realized by developing a tissue adhesive sticking the engineered composite to native tissue.

\section{What was the turning point in your career?}

After graduating with a $\mathrm{PhD}$ degree, I got an opportunity to work in Dr. Ram Mahato's lab at the University of Tennessee Health Science Centre, U.S.A, where I had the chance to learn and devote myself to gene therapy research. Later, I joined Dr. Jennifer Ellisseff's group at Johns 
Hopkins University, U.S.A., where I started to work on cartilage tissue engineering. During these two periods of time, I learned a lot in cellular and molecular biology and molecular pharmaceutics; thus, I moved from being a scientist with purely polymeric background to a "true" biomaterialist/ tissue engineer working in regenerative medicine.

\section{Who has most influenced your research career?}

Besides the two professors mentioned in the previous question, I am very grateful to my $\mathrm{PhD}$ supervisor, Professor Feng Linxian from Zhejiang University, China. I have learnt a lot from Professor Feng in more than knowledge. Her quick-mindedness, ability of prioritization, and strong determination and perseverance have greatly influenced me and nurtured me from a student to a scientist.

\section{What is your philosophy of educating graduate students?}

Besides the delivery of scientific knowledge in the specialty area, training for graduate students must also focus on various skills and abilities, such as verbal communication and logical analysis. With this kind of training, they would be able to solve problems independently and learn new things on their own more effectively in their future research and life. In some sense, I also think that students' maturity and emotional quotient (EQ) are even more important than their technical skills and knowledge as these contribute to their character development toward a more responsible and successful scientist.

Pharmaceutical scientists are faced with the dilemma of having to publish in biomedical or basic science journals. Does this mean cutting-edge science will not likely be featured in journals like Pharmaceutical Research?

That depends on the definition of "cutting edge." I think a lot of pharmaceutical scientists are developing new and immediate solutions that are practical and helpful to benefit human health. Pharmaceutical Research has been publishing these excellent works. In this sense, these are considered as "cutting edge." Especially in the recent decade, molecular pharmaceutics have become one of the main streams of research, and more cutting-edge science/ knowledge will be discovered and published in journals like Pharmaceutical Research.

Where are the fields of biomaterials and tissue engineering going, and how do the articles in this theme issue fill the gap?

In the recent decades, the term "drug" is no longer restricted to chemical molecules only. Biomacromolecules like DNA and siRNA are also common "drugs" that are delivered. As biological molecules are highly susceptible to environmental changes, the main challenge in delivery of these molecules lies in development of a carrier capable of both specific targeting and prolonging sustained delivery in vivo.

In tissue engineering, stem cell is one of the therapeutic cells to be delivered. However, it remains hard to handle autologous stem cells for therapeutic purposes; furthermore, finding a suitable cell carrier system that could support both stem cell viability and differentiation is yet another challenge. Hence, I believe the upcoming research directions would likely be geared toward overcoming these limitations.

In this theme issue, the focus is on the current approaches in delivering two groups of therapeutic agents: biomacromolecules and cells. The articles in this issue include various strategies and platform designs for delivering the therapeutic agents mentioned, providing an up-todate status of the available techniques to achieve targeted delivery. In addition, in vitro phenotype-preserving cell expansion methods are also discussed in some articles.

What are the challenges for developing engineered biomaterials for drug/nucleic acid delivery and tissue engineering, and how can they be overcome?

One of the main challenges for research in biomaterials or tissue engineering is the gap between research and clinical or practical application. More communication or cooperation should be developed between research scientists and clinicians or pharmacists.

One example is about how to control the long-term fate of biomaterials, including the control over the degradation process and the ultimate clearance of the biomaterials from the body. The engineered biomaterials should ideally be a transient support, cleared or replaced with neo-tissues after serving their supporting function. Biomaterials should be able to guide a bioresponsive process for ultimate scaffold-free tissue formation. Strategies are yet to be developed and require more thorough understanding of related physiology, pathology, pharmacology, and clinical medicine. Communication and cooperation with clinicians or pharmacists would greatly expedite the understanding and product development process.

Another example is that for most (not all) tissue regeneration, the engineering of angiogenesis has been one of the greatest challenges. Blood vessels should infiltrate the engineered tissue, and implanted tissue should be able to integrate with the native tissue. This requires thorough understanding of fundamentals of biology and extensive preclinical and clinical trials, where clinicians could contribute significantly. 
What is the key to developing successful collaborative relationships between biomaterials/drug delivery scientists like you and more applied pharmaceutical/formulation scientists who can help in product development?

Following the previous question, I think there is no absolute distinction between biomaterial/drug delivery sciences and pharmaceutical/formulation sciences. Both of them are dedicated to finding out an optimal media for administration of a certain medication. The media could be the materials or methodology. The key to develop successful collaborative relationships is that the scientists should be open-minded and communicate more so that product development would be expedited.

What is the place for collaboration with industry and academia in Singapore and other major Asian countries like Japan, South Korea, China, and India?

The Singapore Economic Development Board (EDB) and Agency for Science, Technology, and Research (A*STAR) have been actively encouraging more collaborations between academia and industry in Singapore. At the same time, they have also been working very hard to expand the communication and cooperation with overseas countries.

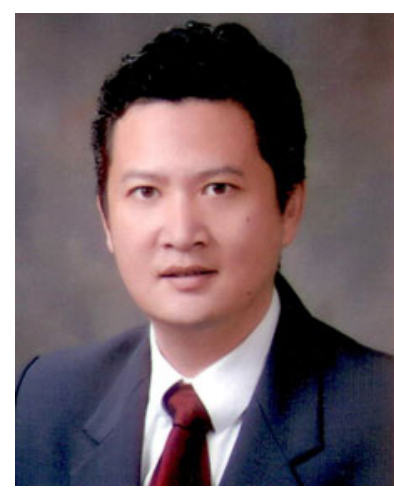

Dr. Dong-An Wang is an Associate Professor of Bioengineering in the School of Chemical and Biomedical Engineering (SCBE), Nanyang Technological University (NTU) in Singapore. He received his B.S. (1997) in Polymer Engineering and his Ph.D. (2001) in Polymer Science from Zhejiang University, China. He was a postdoctoral fellow, in turn, at the University of Tennessee Health Science Centre and Johns Hopkins University in the United States prior to joining NTU faculty in 2005. His research focuses on engineered regenerative medicine in cartilage and bones with specialties in bioresponsive biomaterials for therapeutic cell delivery, gene/antisense delivery for regenerative medicine, adjacent reproducible tissue-sourced stem cells for tissue regeneration, and tissue-biomaterial integration for in situ tissue engineering. Dr. Wang has authored over 70 publications, one book chapter, and four patents (issued or pending), some of which are either editorially quoted or featured as cover stories in reputable research journals. He has also served as an invited guest editor for a theme issue in Advanced Drug Delivery Reviews (2010). Besides scientific research, Dr. Wang is actively working on higher education in biomaterials and tissue engineering areas and was awarded the Nanyang Award of Excellence in Teaching (2008). Dr. Wang currently serves as an Associate Chair in charge of Graduate Studies in SCBE, NTU. 